\title{
AN AXIOMATIC PROOF OF STIEFEL'S CONJECTURE
}

\author{
JOHN D. BLANTON AND CLINT MCCRORY
}

\begin{abstract}
Stiefel's combinatorial formula for the Stiefel-Whitney homology classes of a smooth manifold is proved, by verifying that the classes defined by his formula satisfy axioms which characterize the Stiefel-Whitney classes.
\end{abstract}

1..Introduction. In [2] there were presented axioms for the homology duals to the Stiefel-Whitney classes of smooth manifolds. We show here that the homology classes defined by the combinatorial formula of Stiefel [7, p. 342] satisfy these axioms.

Halperin and Toledo published the first detailed proof of Stiefel's conjecture [5]. Earlier proofs were outlined by Whitney [9] and by Cheeger [3]. A proof for mod 2 homology manifolds, using Steenrod operations, was found by Ravenel and McCrory (unpublished). An axiomatic proof for mod 2 homology manifolds has been given recently by $L$. Taylor [8], using the method of [2] and a classifying space of Quinn.

Let $\mathfrak{N}$ be the category whose objects are $C^{\infty}$ separable Hausdorff manifolds (without boundary) and whose morphisms are open embeddings, that is $f: M \rightarrow N$ is a morphism of $\mathfrak{R}$ if $M$ and $N$ are objects of $\mathfrak{R}$ and $f$ is a diffeomorphism of $M$ onto an open subset of $N$.

Let $\bar{H}_{*}$ be the homology functor defined using infinite (but locally finite) chains, either singular or simplicial. $\bar{H}_{*}(\cdot ; \mathrm{Z} / 2)$ is a contravariant functor on the category $\Re$, since an open embedding $f: M \rightarrow N$ induces a restriction homomorphism [2]

$$
f^{*}: \bar{H}_{*}(N ; \mathbf{Z} / 2) \rightarrow \bar{H}_{*}(M ; \mathbf{Z} / 2) \text {. }
$$

The total Stiefel homology class

$$
W^{\prime}(M)=W_{0}^{\prime}(M)+W_{1}^{\prime}(M)+\cdots+W_{m}^{\prime}(M)
$$

where $m$ is the dimension of $M$, satisfies the following axioms:

(1) For every $M \in \operatorname{Obj}(\Re)$ and every integer $i, 0<i<m$, there is a Steifel homology class $W_{i}^{\prime}(M) \in \bar{H}_{m-i}(M ; \mathbf{Z} / 2)$.

(2) If $f: M \rightarrow N$ is a morphism of $\mathfrak{N}$, then $f^{*} W^{\prime}(N)=W^{\prime}(M)$.

(3) $W^{\prime}(M \times N)=W^{\prime}(M) \times W^{\prime}(N)$.

(4) For every nonnegative integer $i$ there exists a positive even integer

Received by the editors April 11, 1978 and, in revised form, January 19, 1979.

AMS (MOS) subject classifications (1970). Primary 57D20, 55F40; Secondary 57C99.

Key words and phrases. Stiefel-Whitney class, $C^{\infty}$ manifold, triangulation, barycentric subdivision.

(c) 1979 American Mathematical Society n002-9939/79/0000-0571/\$02.50 
$m>i$ such that

$$
W_{i}^{\prime}\left(P_{m}(\mathbf{R})\right)=\left(\begin{array}{c}
m+1 \\
i
\end{array}\right) x^{\prime}
$$

Here $P_{m}(\mathbf{R})$ is the real projective space of dimension $m$ and $x^{i}$ is the unique nonzero element in $\bar{H}_{m-i}\left(P_{m}(\mathbf{R}) ; \mathbf{Z} / 2\right)$.

In [2] it is proved that there exists a unique homology class $W^{\prime}(M)$ for each $M \in O b j(\mathfrak{N})$ such that the axioms (1)-(4) are satisfied.

Following Halperin and Toledo [5], we let $(K, \varphi)$ denote a smooth triangulation of $M$, and let $K^{\prime}$ denote the first barycentric subdivision of $K$. An infinite simplicial $k$-chain on $M$ will mean a formal infinite sum $\Sigma \lambda_{\sigma} \sigma$ where $\sigma$ is a $k$-simplex of $K^{\prime}$ and $\lambda_{\sigma} \in Z / 2$. These chains form a complex $C_{*}(M)$ from which $\bar{H}_{*}(M ; \mathbf{Z} / 2)$ is defined.

Stiefel [7] conjectured that the infinite chain $s_{k}(M)$ which is the sum of all the $k$-simplexes of $K^{\prime}$ represents the Stiefel homology class $W_{n-k}^{\prime}(M)$.

We will see below that the chains $s_{k}(M)$ are cycles, so their homology classes satisfy axiom (1). (This was proved by Akin [1] and by Halperin and Toledo [5].) Since Halperin and Toledo [6], Milnor, and others have shown that Stiefel's combinatorial classes satisfy axiom (3), we prove only that these classes satisfy axioms (2) and (4).

REMARK. Taylor [8] does not prove axiom (2) (his axiom (A1)) for the combinatorial Steifel-Whitney classes! On the other hand, he shows that axiom (4) can be replaced by simpler axioms (his axioms (A3)-(A6)).

2. Axiom (2) is satisfied. If $M$ is a triangulated PL $m$-manifold with boundary, let $s_{k}(M)$ be the sum of all the $k$-simplexes in the first barycentric subdivision of $M$.

Lemma 1 (CF. [1, Proposition 1(b)]). $\partial s_{k}(M)=s_{k-1}(\partial M)$.

Proof. Let $\alpha=\left\langle\hat{\sigma}_{0}, \ldots, \hat{\sigma}_{k-1}\right\rangle$ be a $(k-1)$-simplex in the first barycentric subdivision, where $\sigma_{0}<\cdots<\sigma_{k-1}$ are simplexes in the given triangulation, and $\hat{\sigma}_{i}$ is the barycenter of $\hat{\sigma}_{i}$. The coefficient of $\alpha$ in $s_{k}(M)$ is the mod 2 Euler number of $\operatorname{Link}\left(\sigma_{k-1}\right)$ (cf. [1, p. 342]). If $\alpha \subset$ Int $M=M \backslash \partial M$ then $\operatorname{Link}\left(\sigma_{k-1}\right)$ is a sphere. If $\alpha \subset \partial M$ then $\operatorname{Link}\left(\sigma_{k-1}\right)$ is a disc.

Let $W_{i}^{\prime}(M) \in \bar{H}_{m-i}(M, \partial M ; Z / 2)$ be the class of $s_{m-i}(M)$.

Proposition 1 (CF. [1, Proposition 2]). If $f: M \rightarrow N$ is a PL homeomorphism of triangulated $P L$ manifolds, $f_{*} W_{i}^{\prime}(M)=W_{i}^{\prime}(N)$.

Proof. Let $M_{f}$ be the mapping cylinder of $f . M_{f}$ is a PL manifold with $\partial M_{f}=M \cup N$. The given triangulations of $M$ and $N$ can be extended to a triangulation of $M_{f}$. Thus, by the lemma, $s_{k}(M)$ and $s_{k}(N)$ are homologous in $M_{f}$. Let $r: M_{f} \rightarrow N$ be the canonical homotopy equivalence. Since $r \mid M=f$, $f_{*} W_{m-k}^{\prime}(M)=W_{m-k}^{\prime}(N)$.

Therefore, by the Whitehead triangulation theorem, we get a well-defined class $W_{i}^{\prime}(M) \in \bar{H}_{m-i}(M, \partial M ; \mathbf{Z} / 2)$ for any smooth $m$-manifold $M$ with 
boundary. Let $W_{i}(M) \in H^{i}(M ; \mathrm{Z} / 2)$ be the Poincaré dual class, and let

$$
W(M)=W_{0}(M)+W_{1}(M)+\cdots+W_{m}(M) \in H^{*}(M ; \mathbf{Z} / 2) .
$$

Proposition 2. (1) If $f: M \rightarrow N$ is a diffeomorphism, $f^{*} W(N)=W(M)$,

(2) $W(\operatorname{Int} M)=W(M) \mid \operatorname{Int} M$,

(3) $W(\partial M)=W(M) \mid \partial M$.

Proof. (1) follows from Proposition 1, and (3) from the lemma. For (2), embed $M$ in the thickened manifold $M_{1}=M \cup(\partial M \times[0, \infty))$. Clearly $W(M)=W\left(M_{1}\right) \mid M$. Applying (3) to the manifold ((Int $\left.\left.M\right) \times\{0\}\right) \cup\left(M_{1} \times\right.$ $(0,1])$, the conclusion follows.

By definition of the map $f^{*}$ in homology, axiom (2) for $W^{\prime}$ is a corollary of the following theorem.

THEOREM 1. If $f: M \rightarrow N$ is a diffeomorphism of $M$ onto an open subset of $N$, then $f^{*} W(N)=W(M)$.

Proof. By (1) of Proposition 2 we can assume $f$ is an inclusion. By (2) we can assume $M$ and $N$ have no boundary. Applying (3) to the manifold $(M \times\{0\}) \cup(N \times(0,1])$, the conclusion follows.

Properties (1)-(3), the proof of (2), and the proof of the theorem are taken from unpublished notes of John Milnor.

3. Axiom (4) is satisfied. Let

$$
\Sigma^{m}=\left\{\left(x_{1}, \ldots, x_{m+1}\right) \in \mathbf{R}^{m+1}|| x_{1}|+\cdots+| x_{m+1} \mid=1\right\},
$$

a polyhedral $m$-sphere. $\Sigma^{m}$ has a canonical triangulation whose vertices are the intersections of $\Sigma^{m}$ with the coordinate axes. Radial projection of $\Sigma^{m}$ onto

$$
S^{m}=\left\{\left(x_{1}, \ldots, x_{m+1}\right) \in \mathbf{R}^{m+1} \mid\left(x_{1}\right)^{2}+\cdots+\left(x_{m+1}\right)^{2}=1\right\}
$$

gives a smooth triangulation of the standard $m$-sphere. Let $\Pi^{m}$ be the cell complex obtained from $\Sigma^{m}$ by identifying $x$ with $-x$ for all $x \in \Sigma^{m}$. Then the first barycentric subdivision $\Pi^{\prime m}=K$ is a simplicial complex which gives a smooth triangulation $(K, \phi)$ of real projective $m$-space $P_{m}(\mathbf{R})$.

Proposition 3. $W_{i}^{\prime}\left(P_{m}(\mathbf{R})\right)$ is represented by the sum of all the $(m-i)$ simplexes of the triangulation $K$.

Proof. By definition, $W_{i}^{\prime}\left(P_{m}(\mathbf{R})\right)$ is represented by the sum of all the $(m-i)$-simplexes of the first barycentric subdivision $K^{\prime}$. Although $K$ is not the barycentric subdivision of a triangulation, it is the barycentric subdivision of the regular cell complex $\Pi^{m}$ whose cells are simplexes. The arguments of Lemma 1 and Proposition 1 apply without change to show that $s_{m-i}(K)$ is homologous to $s_{m-i}\left(\Pi^{m}\right)$.

RemarK. This proposition also follows from [6, Proposition (i), p. 243].

Each $k$-cell of the $m$-sphere $\Sigma^{m}$ lies in a $(k+1)$-dimensional linear subspace of $\mathbf{R}^{m+1}$ defined by the vanishing of $m-k$ coordinates of $\mathbf{R}^{m+1}$. For 
each such linear subspace $\mathbf{R}_{j}^{k+1}$, let

$$
\Sigma^{m} \cap \mathbf{R}_{j}^{k+1}=\Sigma_{j}^{k}
$$

and let $\Pi_{j}^{k}$ be the image of $\Sigma_{j}^{k}$ in $\Pi^{m}$. There are

$$
\left(\begin{array}{c}
m+1 \\
k+1
\end{array}\right)=\left(\begin{array}{l}
m+1 \\
m-k
\end{array}\right)
$$

such $k$-dimensional projective subspaces $\Pi_{j}^{k}$ in $\Pi^{m}$.

Let $t_{k}\left(\Pi^{m}\right)$ be the chain of $k$-simplexes of $K$ which are not in the barycentric subdivision of some $\Pi_{j}^{k}$.

Proposition 4. The chain $t_{k}\left(\Pi^{m}\right)$ is a boundary, $0<k<m$.

Proof. We shall show that $t_{k}\left(\Pi^{m}\right)$ is the sum of an even number of mutually homologous $k$-cycles. Each simplex of $t_{k}\left(\Pi^{m}\right)$ is contained in a unique $k$-dimensional projective subspace $\Lambda$ of $P_{m}(R)$. We will see that $\Lambda$ is a subcomplex of $K$. Thus if $c(\Lambda)$ is the sum of all the $k$-simplexes of $\Lambda$, then $c(\Lambda)$ is a cycle representing the generator of $H_{k}\left(P_{m}(\mathbf{R}) ; \mathrm{Z} / 2\right)$. Furthermore, the $k$-simplexes of $\Lambda$ all belong to $t_{k}\left(\Pi^{m}\right)$, so $t_{k}\left(\Pi^{m}\right)$ is the sum of all the cycles $c(\Lambda)$ determined in this way. Finally we will show that there are an even number of such cycles $c(\Lambda)$.

Let $\sigma$ be a simplex of $t_{k}\left(\Pi^{m}\right)$, and let $s$ be one of the two $k$-simplexes of the barycentric subdivision of $\Sigma^{m}$ which correspond to $\sigma$. Let $L$ be the $(k+1)$ dimensional linear subspace of $\mathbf{R}^{m+1}$ containing $s$. The image of $L \cap S^{m}$, under the canonical map $S^{m} \rightarrow P_{m}(\mathbf{R})$, is the subspace $\Lambda$ determined by $\sigma$.

For $i=1, \ldots, m+1$, let $\pm v_{i}$ be the vertex of $\Sigma^{m}$ corresponding to $\pm e_{i}$, where $e_{i}$ is the $i$ th standard basis vector of $\mathbf{R}^{m+1}$. Then the barycentric coordinate corresponding to $\pm v_{i}$ in $\Sigma^{m}$ is $\pm x_{i} \mid \Sigma^{m}$, where $x_{i}$ is the $i$ th coordinate function of $\mathbf{R}^{m+1}$.

Let $S$ be the simplex of $\Sigma^{m}$ which carries $s$. Since $s$ is not in the barycentric subdivision of the $k$-skeleton of $\Sigma^{m}$, we have $\operatorname{dim} S>k$. Let $I=\left\{i \mid \pm v_{i}\right.$ is a vertex of $S\}$, and define $\varepsilon: I \rightarrow\{+1,-1\}$ so that $\varepsilon(i) v_{i}$ is a vertex of $S$ for each $i \in I$. Now each vertex $w$ of $s$ is the barycenter of some face $T(w)$ of $S$. Let $w_{1}, \ldots, w_{k+1}$ be the vertices of $s$, ordered so that $T\left(w_{i}\right)$ is a face of $T\left(w_{j}\right)$ for $i<j$. Define a partition $J=\left\{J_{0}, \ldots, J_{k+1}\right\}$ of the set $\{1, \ldots, m+1\}$ as follows. Let $J_{0}=\{1, \ldots, m+1\}-I, J_{1}=\left\{i \in I \mid \varepsilon(i) v_{i}\right.$ is a vertex of $\left.T\left(w_{1}\right)\right\}$, and for $p=2, \ldots, k+1$, let $J_{p}=\left\{i \in I \mid \varepsilon(i) v_{i}\right.$ is a vertex of $T\left(w_{p}\right)$ but not of $\left.T\left(w_{p-1}\right)\right\}$. A dimension count shows that the subspace $L$ of $\mathbf{R}^{m+1}$ spanned by the vertices of $s$ is given by the equations

$$
\begin{cases}x_{i}=0, & i \in J_{0}, \\ \varepsilon(i) x_{i}=\varepsilon(j) x_{j}, & i, j \in J_{p}, p=1, \ldots, k+1 .\end{cases}
$$

Let $\left|J_{p}\right|$ denote the number of elements of $J_{p}$. We have $\left|J_{0}\right|=(m+1)-$ $(\operatorname{dim} S+1)<m-k$. A partition $J=\left\{J_{0}, \ldots, J_{k+1}\right\}$ of $\{1, \ldots, m+1\}$ 
with $\left|J_{0}\right|<m-k$ will be called allowable. Any allowable partition $J$ of $\{1, \ldots, m+1\}$, together with a function $\varepsilon: J_{1} \cup \cdots \cup J_{p} \rightarrow\{+1,-1\}$, defines a $(k+1)$-dimensional subspace $L$ of $\mathbf{R}^{m+1}$ by the equations (*). The set $L \cap \Sigma^{m}$ is a subcomplex of the barycentric subdivision $\Sigma^{\prime m}$, so the corresponding space $\Lambda \subset P^{m}(\mathbf{R})$ is a subcomplex of $K$. Since $\left|J_{0}\right|<m-k$, each simplex of $L \cap \Sigma^{\prime m}$ is carried by a simplex $S$ of $\Sigma^{m}$ with $\operatorname{dim} S>k$, so all the $k$-simplexes of $\Lambda$ belong to $t_{k}\left(\Pi^{m}\right)$, as desired.

It remains to show that there are an even number of such projective subspaces $\Lambda$. For each allowable partition $J$ there is at least one $p$ such that $\left|J_{p}\right|>1$, since $\left|J_{0}\right|<m-k$. For each allowable $J$, choose such a $p$, and choose $i_{0} \in J_{p}$. For each $\Lambda$ corresponding to the partition $J$ and the function $\varepsilon$, let $\Lambda^{\prime}$ be the subspace corresponding to $J$ and the function $\varepsilon^{\prime}$ defined by $\varepsilon^{\prime}(i)=\varepsilon(i)$ for $i \neq i_{0}$ and $\varepsilon^{\prime}\left(i_{0}\right)=-\varepsilon\left(i_{0}\right)$. Then $\Lambda^{\prime} \neq \Lambda$ and $\left(\Lambda^{\prime}\right)^{\prime}=\Lambda$. The existence of this involution $\Lambda \mapsto \Lambda^{\prime}$ shows that there are an even number of subspaces $\Lambda$ corresponding to each allowable partition $J$, so there are an even number of $\Lambda$ in all.

\section{THEOREM 2. $W_{i}^{\prime}\left(P_{m}(\mathbf{R})\right)=\left(\begin{array}{c}m+1 \\ i\end{array}\right) x^{\prime i}$.}

Proof. Let $k=m-i$. By Proposition 3, $W_{i}^{\prime}\left(P_{m}(\mathbf{R})\right)$ is represented by $s_{k}\left(\Pi^{m}\right)$, the sum of all the $k$-simplexes in the barycentric subdivision $\Pi^{\prime m}=$ $K$. Let $s_{k}\left(\Pi_{j}^{k}\right)$ be the sum of all the simplexes of $K$ in $\Pi_{j}^{k}$. Since $\Pi_{j}^{k}$ is a $k$-dimensional projective space, $s_{k}\left(\Pi_{i}^{k}\right)$ represents the generator $x^{i}$ of $H_{k}\left(P_{m}(\mathbf{R}) ; \mathrm{Z} / 2\right)$. But

$$
s_{k}\left(\Pi^{m}\right)=\sum_{j=1}^{l} s_{k}\left(\Pi_{j}^{k}\right)+t_{k}\left(\Pi^{m}\right), \quad l=\left(\begin{array}{c}
m+1 \\
i
\end{array}\right),
$$

and $t_{k}\left(\Pi^{m}\right)$ is homologous to zero by Proposition 4 .

Theorem 2 implies axiom (4) for all integers $i<m$. Theorem 2 has been proved independently by Goldstein and Turner [4].

\section{REFERENCES}

1. E. Akin, Stiefel-Whitney homology classes and bordism, Trans. Amer. Math. Soc. 205 (1975), 341-359.

2. J. Blanton and P. Schweitzer, S.J., Axioms for characteristic classes of manifolds, Proc. Sympos. Pure Math., vol. 27, Amer. Math. Soc., Providence, R. I., 1975, pp. 436-443.

3. J. Cheeger, A combinatorial formula for Stiefel-Whitney classes, Topology of Manifolds, J. C. Cantrell and C. H. Edwards (eds.), Markham Publishing Co., Chicago, Ill., 1970, pp. 470-471.

4. R. Goldstein and E. C. Turner, Stiefel-Whitney homology classes of quasi-regular cell complexes, Proc. Amer. Math. Soc. 64 (1977), 157-162.

5. S. Halperin and D. Toledo, Stiefel-Whitney homology classes, Ann. of Math. 96 (1972), 511-525.

6. The product formula for Stiefel-Whitney homology classes, Proc. Amer. Math. Soc. 48 (1975), 239-244. 
7. E. Stiefel, Richtungeffelder und Fernparallelismus in n-dimensonalen Manigfaltigkeiten, Comment. Math. Helv. 8 (1936), 305-353.

8. L. Taylor, Stiefel-Whitney homology classes, Quart. J. Math. Oxford Ser. (2) 28 (1977), 381-387.

9. H. Whitney, On the theory of sphere bundles, Proc. Nat. Acad. Sci. U.S.A. 26 (1940), 148-153.

Department of Mathematics, St. John Fishigr Collbob, Rochestre, New York 14618

Departmant of Mathematics, Brown Universtty, Providence, Rhode Island 02912 